Sieverding M (1990) Psychologische Barrieren in der beruflichen Entwicklung von Frauen. Das Beispiel der Medizinerinnen. Enke, Stuttgart

Statistisches Bundesamt (2000, 2003, 2005). Bildung und Kultur Fachserie 11. Wiesbaden

Uhlenberg P, Cooney TM (1990) Male and female physicians: Family and career comparison. Soc

Sci Med 30: 373-378

Ward A (1982) Careers fo medical women. Br Med J 284: 31-33

Wetterer A (1995) Die soziale Konstruktion von Geschlecht in Professionalisierungsprozessen.

Campus, Frankfurt

Wirth H, Dümmler K (2004) Zunehmende Tendenz zu späteren Geburten und Kinderlosigkeit

bei Akademikerinnen. Eine Kohortenanalyse auf der Basis von Mikrozensusdaten. Informationsdienst Soziale Indikatoren (ISI) 32: 1-10

Zuber MA (2001) Analyse des Frauenanteils bei verschiedenen Qualifikationsstufen des Fachgebietes Humanmedizin in Deutschland. Dtsch Med Wschr 126: 65-71

\title{
1.3 Psychologische Karrierehindernisse im Berufsweg von Frauen
}

Monika Sieverding

\subsubsection{Welche Widerstände verhindern berufliches Engagement und beruflichen Erfolg von Frauen?}

Obwohl immer mehr Frauen sich für den Medizinerberuf entscheiden, erreicht nach wie vor »nur ein Bruchteil des Ausgangspotentials eine Führungsposition « (Bund-Länder-Kommission 2004; Kap. 1.2). Dies gilt nicht nur für Medizinerinnen, sondern für Frauen in den verschiedensten Berufsfeldern. Welche Widerstände sind es, die sich Frauen bei der Verfolgung ihrer beruflichen Ziele in den Weg stellen? Auf Lewin und seine »topologischen Psychologie (1936, dt. Fassung 1969) geht der Begriff der »Barriere« zurück: »Eine Person verfolgt ein bestimmtes Ziel, jedoch ist es gegenwärtig für sie schwierig oder gar unmöglich, es zu erreichen ... eine Barriere (B) macht die Lokomotion von P (Person) nach Z (Ziel) schwierig oder verhindert sie völlig« (Lewin 1969). Folgt man diesem Modell, wird schnell klar, dass die berufliche Entwicklung von Frauen nicht durch eine einzige, sondern durch eine Vielzahl von Barrieren behindert wird.

Die äußeren oder sozialen Barrieren in der beruflichen Entwicklung von Frauen sind weitgehend bekannt. Dies sind in Deutschland beispielsweise steuerliche Rahmenbedingungen, die insbesondere die Teilzeitberufstätigkeit von verheirateten Frauen unattraktiv machen (bekannt als »negativer Anreiz des Ehe- 
gattensplitting «) 1 . Für Ärztinnen erweisen sich überholte Mutterschutzrichtlinien als schwerwiegende Karrierehemmnisse (BLK 2004). Ein anderer Tatbestand ist ein so weitgehender Mangel an Kinderbetreuungseinrichtungen und Ganztagesschulen, der geradezu auf eine gesamtgesellschaftliche Verweigerung schließen lässt (Zahlen zum Nichtvorhandensein von Kindergärten an Medizinischen Fakultäten; BLK 2004).

Offene Ausschlüsse und Diskriminierungen von Frauen - z. B. aus bestimmten beruflichen Positionen - sind heutzutage nicht mehr zulässig. Allerdings gibt es nach wie vor subtile Formen der Diskriminierung von Frauen bei Einstellungen, Beförderungen und sonstigen Förderungen. Für Aufruhr gesorgt hat beispielsweise die in der renommierten Zeitschrift "Nature « veröffentlichte Studie von Wenneras \& Wold (1997), in der zweifelsfrei nachgewiesen wurde, dass in Schweden die Anträge von Wissenschaftlerinnen auf Forschungsförderung bei gleicher objektiver Leistung signifikant schlechtere Chancen auf Förderung hatten als die Anträge von männlichen Kollegen. Bezeichnend wurde dieser Artikel mit der Überschrift »Nepotism (Vetternwirtschaft) and Sexism in Peer Review« betitelt. Eine Studie des Massachusetts Institute of Technology in den USA 1999 erbrachte, dass Wissenschaftlerinnen deutlich schlechtere Arbeitsbedingungen und weniger Förderung erhielten als ihre männlichen Kollegen (Massachusetts Institute of Technology 1999).

Die beruflichen Karrierechancen von Frauen werden jedoch nicht nur durch äußere Faktoren, sondern auch durch psychologische Barrieren beeinträchtigt. Als solche sind beispielsweise ein typisch feminines Geschlechtsrollenselbstkonzept, eine starke Identifikation mit der Mutter-Kind-Ideologie oder eine zu bescheidene Selbsteinschätzung und Selbstdarstellung von Frauen zu nennen. Dabei darf nicht vergessen werden, dass solche »inneren « Barrieren nicht unabhängig von »äußeren « Barrieren entstehen (Sieverding 1990). Sie können interpretiert werden als Reaktion auf äußere Barrieren, als verinnerlichte gesellschaftliche Erwartungen, als Kompromissbildung zwischen äußeren Anforderungen und inneren Bedürfnissen. Sie sind nicht einfach von vornherein »da «, sondern entwickeln sich im Verlauf der geschlechtsspezifischen Sozialisation und Erfahrung im Elternhaus, in der Schule, in der Universität und im Berufsleben. Da sie weitgehend erlernt sind, können sie - nach den Regeln der Lernpsychologie - auch wieder verlernt werden. Dazu ist jedoch eine vorherige Bewusstmachung unabdingbar.

1 siehe dazu die Berechnung von Schmid (2003, Folie 12): Wenn ein Ehemann 40.000 Euro verdient und die Ehefrau nicht berufstätig ist, beträgt die gemeinsame Steuerlast 6400 Euro. Verdient die Frau 15.000 Euro »hinzu«, so erhöht sich die gemeinsame Steuerlast auf 11.200 Euro. Von den 15.000 Euro bleiben de facto nur 10.200 Euro mehr. Durch das Ehegattensplitting wird somit das traditionelle Modell der Versorgerehe einseitig gefördert. 
In diesem Artikel wird ein Überblick über einige wichtige psychologische Barrieren gegeben, die alle mehr oder weniger eng mit traditionellen Geschlechterrollen und Geschlechterstereotypen assoziiert sind. Grob vereinfacht, könnte man es so formulieren: Traditionelle Geschlechterrollen und Geschlechterstereotpye sind die Hauptbarrieren für die berufliche Entwicklung von Frauen, die anderen Faktoren können als Subbarrieren verstanden werden.

\subsubsection{Traditionelle Geschlechterrollen und Geschlechterstereotpye}

Geschlechterrollen sind definiert als normative Erwartungen der Gesellschaft, die am biologischen Geschlecht anknüpfen. Die Erwartungen definieren die Arbeitsteilung und die hierarchische Beziehungsstruktur (Machtverteilung) zwischen den Geschlechtern sowie als wünschenswert angesehene Eigenschaften, Interessen und Fähigkeiten, die »der « Mann oder »die" Frau haben soll, je nach herrschendem Leitbild von Männlichkeit und Weiblichkeit (Alfermann 1996).

Bekannt wurde das Modell von Parsons und Bales (1955). Darin wurden Männern und Frauen aufgrund ihres biologischen Geschlechts unterschiedliche Geschlechterrollen in den Systemen Familie und Gesellschaft zugewiesen. An den Mann wurden Verhaltenserwartungen entsprechend der instrumentellen oder aufgabenbezogenen Rolle gerichtet. Er galt als zuständig für die Beziehungen der Familie nach außen (vor allem für ihre materielle Basis); innerhalb der Familie sollte er die letzte Entscheidungsinstanz sein, Bestrafungen ausführen und auf Einhaltung von Disziplin und Kontrolle achten. Als für einen Mann typisch und angemessen galten psychologische Charakteristika, die ihn für diese Rolle befähigen: Unabhängigkeit, logisches Denken, Wettbewerbsorientierung und Abenteuergeist (später auch als »instrumentelle« Eigenschaften bezeichnet). An die Frau wurden entsprechend der expressiven Rolle komplementäre Verhaltenserwartungen gerichtet. Sie wurde als zuständig angesehen für die inneren Angelegenheiten in der Familie, vor allem für die sozialemotionale Unterstützung aller Familienangehörigen. Weiterhin wurde ihr eine Vermittlerrolle zugeschrieben, ihre Aufgabe sollte es sein, ein harmonisches Gleichgewicht in der Familie zu schaffen. Von ihr wurden folgende psychologischen Charakteristika erwartet: Wärme, Einfühlsamkeit, Emotionalität und die Sorge um andere (später als »expressive« Eigenschaften bezeichnet).

Natürlich haben sich seit den 50er Jahren die Inhalte der Geschlechterrollen geändert (Abele 1997; Alfermann 1996). Angestoßen durch die Frauenbewegung der 70er und 80er Jahre wurde insbesondere die traditionelle Frauenrolle massiv in Frage gestellt und »neue Männer " gefordert. Und tatsächlich ist die Quote der berufstätigen Frauen - vor allem der Frauen mit Kindern - seitdem gestiegen, und inzwischen beteiligen sich auch Väter an Haushalt und Kindererziehung. Es gibt 
jedoch vor allem in Westdeutschland eine große Diskrepanz zwischen Anspruch und Wirklichkeit, gerade was die Rollenverteilung in der Familie betrifft. Werden Männer und Frauen nach ihren Einstellungen dazu befragt, befürworten sie mit großer Mehrheit das Partnerschaftsmodell, wonach die Aufgaben in der Familie gleichberechtigt zwischen Mann und Frau verteilt werden sollten und beide die Möglichkeit haben sollten, berufstätig zu sein. Tatsache ist jedoch, dass in sehr vielen Fällen nach der Geburt des ersten Kindes, noch stärker aber nach der Geburt des zweiten Kindes wieder ein traditionelles Modell gelebt wird (Sieverding 1990 und 1992).

Geschlechterstereotpye als vereinfachte Bilder in unserem Kopf sind Annahmen über typische Eigenschaften und Charakteristika von Männern und Frauen. Sie beinhalten eine deskriptive und eine normative Komponente, d. h.: Welche typischen Eigenschaften hat ein Mann/eine Frau? Welche Eigenschaften sollte ein Mann/eine Frau haben? Und, darüber hinaus, nicht zu vergessen: Welche Eigenschaften sollte ein Mann/eine Frau nicht haben? Die empirische Erfassung solcher Vorstellungen begann in den USA in den 60er Jahren des letzten Jahrhunderts, so ermittelten beispielsweise Rosenkrantz und Mitarbeiter (1968) Geschlechterstereotpye von amerikanischen College-Studierenden. Sie fanden damals, dass es mehr typische und mehr positive Eigenschaften gab, die mit dem männlichen Geschlecht assoziiert wurden. Nach Alfermann (1996) ist dieses Ergebnis darauf zurückzuführen, dass das männliche Geschlecht als die dominante Gruppe (in der amerikanischen Gesellschaft) auch die Bewertung der Stereotype beeinflusst. Seit dieser »Pionierstudie" der Geschlechterstereotpyenforschung wurde eine Vielzahl von weiteren Untersuchungen über Männlichkeits- und Weiblichkeitsklischées durchgeführt. Bekannt geworden ist beispielsweise die kulturvergleichende Studie von Williams und Best (1990), in der sich herausstellte, dass Geschlechterstereotpye in sehr unterschiedlichen Ländern recht große Übereinstimmungen aufwiesen. In - Tabelle 1.5 sind diejenigen Eigenschaften aufgeführt, die übereinstimmend in allen (25) oder fast allen (24) beteiligten Staaten als typisch männlich bzw. weiblich bezeichnet worden waren.

- Tab. 1.5. Kulturübergreifende Geschlechterstereotpye in den 80er Jahren (Williams u. Best 1990; Übersetzung aus Alfermann 1996)

Stereotype maskuline Eigenschaften

abenteuerlustig robust

aggressiv selbstherrlich

dominant stark

kräftig

kühn

maskulin

\section{unabhängig}

unnachgiebig

unternehmungslustig
Stereotype feminine Eigenschaften liebevoll einfühlsam feminin gefühlvoll träumerisch unterwürfig 
Geschlechterstereotpye haben sich zwar im Lauf der Zeit verändert. Der Kern ist doch im Wesentlichen gleich geblieben. Nach wie vor lässt sich das männliche Geschlechterstereotyp als ein »Cluster von Kompetenz und von Aktivität«, das weibliche Geschlechterstereotyp als ein »Cluster von Emotionalität « (Alfermann 1996) und "Besorgtsein um andere kennzeichnen. Oder anders ausgedrückt: Instrumentelle Eigenschaften kennzeichnen nach wie vor das männliche und expressive Eigenschaften das weibliche Stereotyp.

Die Mehrzahl der bisher vorliegenden Studien zu Geschlechterstereotpyen konzentrierte sich auf die Erfassung von Persönlichkeitsmerkmalen. Eckes (1997) fand in einer Studie, in der er Geschlechterstereotpye über einen Prototypenansatz ermittelte, heraus, dass Persönlichkeitseigenschaften besonders zentrale Bestandteile sind. Daneben spielen aber weitere Komponenten eine wichtige Rolle, z. B. die äußere Erscheinung, Einstellungen und Werte sowie offenes Verhalten. In seiner lesenswerten Studie wurden nicht nur Vorstellungen vom »typischen « Mann und der »typischen« Frau, sondern Substereotype von Männern und Frauen erfasst und charakterisiert. Als weibliche Substereotypen schälten sich vier Frauentypen heraus:

- Cluster A:

- Tussie,

- Schickimicki,

- Sekretärin,

- Spießerin;

- Cluster B:

- Mauerblümchen,

- Naive,

- »typische« Frau,

- Hausfrau;

- Cluster C:

- Selbstbewusste

- Intellektuelle,

- Karrierefrau,

- Dame und

- Cluster D:

- Alternative,

- Feministin,

- Emanze.

Diesen verschiedenen Substereotypen sollten typische Merkmale zugeordnet werden. »Die Emanze « wurde beispielsweise durch folgende Merkmale charakterisiert: Lieblingsfarbe lila, ungeschminkt, trägt selbstgestrickte Pullover, sieht Männer als Unterdrücker, lehnt traditionelle Frauenrolle ab, diskutiert gerne, tritt aktiv für 
Frauenrechte ein und geht auf Demos. Die »Hausfrau « dagegen wurde mit folgenden Merkmalen beschrieben: mollig, trägt bequeme Kleidung, Dauerwelle, opfert sich für Familie auf, stellt eigene Bedürfnisse zurück, möchte ihre Kinder stets umsorgen, verbringt viel Zeit in der Küche und achtet auf Sauberkeit. Die »Feministin « wurde so charakterisiert: aktiv in Frauengruppen, liest Frauenliteratur, politisch links, aggressiv, rechthaberisch, fällt anderen ins Wort. (Diese Assoziationen mögen erklären, warum manche Frauen es als Schimpfwort verstehen, wenn sie von anderen als »Feministin« tituliert werden.) Als Letztes soll die Charakterisierung der »typischen Frau» berichtet werden: Sie besitzt ein gepflegtes Äußeres, hat den Wunsch nach einer Familie und das Bedürfnis nach Sicherheit. Sie zeigt Gefühle, ist verständnisvoll, ist unentschlossen und abhängig (Eckes 1997).

Um zu verstehen, wie Geschlechterstereotpye entstehen, ist die Soziale-RollenTheorie von Alice Eagly (1987) hilfreich. Diese Theorie besagt, dass wir - meist unbewusst - Menschen danach beurteilen, was sie tun: »People are what they do . Wenn wir eine Person dabei beobachten, wie sie ein Kind wickelt und füttert, nehmen wir an, dass sie über fürsorgliche und einfühlsame Qualitäten verfügt. Erleben wir eine andere Person, die einer Gruppe von Angestellten Anweisungen gibt, schließen wir, dass diese Person durchsetzungsfähig und selbstbewusst ist. Die Tatsache, dass in den meisten Gesellschaften die Rolle der Kindererziehung und die Sorge für Haushalt und Familie an Frauen delegiert werden, ist nach Eagly der Grund dafür, dass Frauen mehr expressive (fürsorgliche) Eigenschaften zugeschrieben werden. Da in den meisten Gesellschaften sehr viel mehr Männer Führungspositionen besetzen, wird angenommen, dass Männer mehr instrumentelle Eigenschaften - wie z. B. Durchsetzungsfähigkeit, Selbstsicherheit und Unabhängigkeit - »besitzen«. Die Annahmen der Theorie wurden in einer Vielzahl von Studien überprüft. So konnte beispielsweise gezeigt werden, dass Hausfrauen mehr expressive Eigenschaften als berufstätigen Frauen zugeordnet werden, dagegen wird bei Hausmännern ein geringeres $\mathrm{Maß}$ an instrumentellen Eigenschaften vermutet als bei berufstätigen Männern (Bless et al. 1992).

Deaux und Major (1987) haben ein Modell vorgestellt, in dem sie demonstrieren, wie Geschlechterstereotpye zu sich selbst erfüllenden Prophezeiungen werden können. Sie postulieren darin, dass sich die Geschlechterstereotype in Prozessen der sozialen Interaktion ständig reproduzieren. So habe ich als handelnde Person bestimmte Annahmen darüber, was Frauen im Allgemeinen und ich im Besonderen für Eigenschaften mitbringen. Die Personen, mit denen ich interagiere, z. B. Eltern, Partner, Freunde und Freundinnen, Kollegen, Vorgesetzte, haben ebenfalls Annahmen darüber, wie Frauen im Allgemeinen und ich im Besonderen bin. Je weniger sie mich persönlich kennen, also z. B. neue Kollegen und/oder Vorgesetzte, desto eher werden sie allgemeine Annahmen über Frauen auch auf mich anwenden. Diese Erwartungen werden sie mir in einer konkreten Situation oder bei 
der Besprechung einer Aufgabe mehr oder weniger offen signalisieren, z. B. indem sie sie direkt ansprechen oder lediglich durch nonverbale Signale andeuten. Je nachdem, wie gut ihre Erwartungen zu meinen Erwartungen passen und wie abhängig ich von den Erwartungen dieser anderen Person bin, werde ich tatsächlich ihre Annahmen durch mein Verhalten bestätigen.

Ich möchte die - hier sehr stark vereinfacht dargestellte - Theorie von Deaux und Major (1987) auf ein Beispiel aus dem medizinischen Alltag anwenden:

Professor Schulz, der Chefarzt einer gynäkologischen Abteilung hat zwei neue Assistenzärzte, Frau Schneider und Herrn Müller. Herr Schulz ist der festen Überzeugung, dass Ärztinnen einfühlsamer sind und besser mit Patienten sprechen können. Dagegen hält er Ärzte für analytischer denkend und pragmatischer. Es gibt nun aktuell zwei Aufgaben zu vergeben: Mehrere Patientinnen benötigen (z. B. nach der Übermittlung einer infausten Diagnose) ein ausführliches ärztliches Gespräch, in dem auch auf psychische Aspekte und Probleme eingegangen werden muss. Außerdem soll ein wissenschaftlicher Artikel über eine kürzlich abgeschlossene Studie geschrieben werden. Herr Schulz wird nun Frau Schneider bitten, die Patientinnengespräche zu führen, ihr gleichzeitig signalisierend, dass sie dafür doch »als Frau « die notwendige Einfühlsamkeit und Kommunikationsfähigkeit mitbringe. Die Erstellung des Artikels hingegen vergibt er an Herrn Müller, die Erwartung äußernd, dass dieser den Artikel möglichst zügig erstellen werde. Was passiert nun? Frau Schneider und Herr Müller werden die Aufgaben, wie delegiert, übernehmen (müssen) und sie werden sich voraussichtlich bemühen, ihren Job möglichst gut zu machen. Frau Schneider führt die Patientinnengespräche, Herr Müller schreibt den Artikel. Falls sie Schwierigkeiten bei ihren Aufgaben haben, werden sie sich möglicherweise Hilfe und Unterstützung suchen; die Wahrscheinlichkeit, dass sie ihrem Chef rückmelden, dass sie der Aufgabe nicht gewachsen sind oder dass sie die Aufgabe nicht übernehmen wollen, ist (insbesondere wegen des Abhängigkeitsverhältnisses) recht gering. Wahrscheinlich werden beide ihre Aufgabe "gut " machen. Die erfolgreiche Ausführung der Aufgaben bestätigt nun nicht nur die bestehenden Geschlechterstereotpye ihres Vorgesetzten, sondern auch ihre tatsächlichen Fähigkeiten: Durch jedes weitere »schwierige« Patientinnengespräch werden die empathischen und kommunikativen Fertigkeiten von Frau Schneider verbessert, dagegen steigert sich die wissenschaftliche Kompetenz des Herrn Müller mit jeder neuen Studie und jedem neuen Artikel. Und es geht noch weiter: Da Frau Schneider noch nie einen wissenschaftlichen Artikel formuliert hat, traut sie es sich immer weniger zu, dagegen macht Herr Müller einen großen Bogen um »schwierige» Patientinnen. Nach einiger Zeit ist dann tatsächlich Frau Schneider die »bessere « Ansprechpartnerin für Patientinnen, Herr Müller der »bessere» Wissenschaftler, die Geschlechterstereotpye haben sich verselbständigt und in gewisser Weise bewahrheitet. Ein Rollentausch wird dann - nicht nur aus der Sicht der Klinik immer unwahrscheinlicher und unökonomischer. 
Häufig ist es im Alltag so, dass tatsächlich anfangs (geringfügige) Unterschiede in bestimmten Fähigkeiten bestehen. So haben stillende Mütter unweigerlich einen biologischen Vorteil gegenüber ihren Partnern, wenn es um die Beruhigung eines schreienden Säuglings geht. Die Frage ist, was aus solchen Ausgangsunterschieden abgeleitet wird: Heißt das, dass nun die Mutter die ersten Lebensjahre (oder länger, oder immer?) die Hauptbezugsperson für das Kind sein muss? Oder heißt es vielmehr, dass ein Vater sich besondere Mühe geben sollte, in der Beziehung zum Kind den biologischen Anfangsnachteil wettzumachen? Beobachtungen an Paaren, in denen nicht die Mutter, sondern der Vater die meiste Zeit mit dem Kind zusammen ist, zeigen jedenfalls, dass Väter das Kinder-Beruhigen sehr wohl erlernen können. Eine Kollegin von mir, die nach der Geburt ihres Kindes sehr schnell wieder in den Beruf eingestiegen ist, gibt ihr schreiendes Kleinkind gern an ihren Mann, der ein Erziehungsjahr genommen hat, weiter: »Da, nimm! «

Die Ausführungen verdeutlichen, dass Geschlechterrollen und -stereotype einerseits die Welt vereinfachen, andererseits erschweren. Vereinfachen deshalb, da klar ist, was von wem in einer bestimmten Situation erwartet wird ("Wer entfernt die Spinne? «, »Wer repariert den Rasenmäher? «, »Wer wickelt das Kind? «, »Wer schreibt die Weihnachtsgrußkarten?», "Wer bleibt zu Hause, wenn ein Kind geboren wird? «). Erschweren deshalb, da sich häufig aus zunächst banal anmutenden Zuschreibungen und Arbeitsaufteilungen fest gefügte Rollenmuster und -verhalten (in einer Partnerschaft, in einer Familie, in einer Arbeitsgruppe) zementieren, aus denen dann nur noch schwer auszubrechen ist.

\subsubsection{Weiblichkeit und Karriere: inkompatibel?}

Eine Ursache für die Unterrepräsentanz von Frauen in Leitungspositionen wird darin gesehen, dass es in Beurteilungsprozessen von Frauen zu Vorurteilen und Potentialfehleinschätzungen (insbesondere zu Unterschätzungen) kommt, da Führungsqualifikationen (wie Selbstsicherheit) eher mit dem männlichen als mit dem weiblichen Geschlechterstereotyp assoziiert werden (Eagly \& Karau 2002; Sczesny 2003). Die Rollen-(In-)Kongruitätstheorie von Eagly und Karau (2002) bringt das Dilemma von Frauen auf den Punkt: Nach dieser Theorie nehmen viele Menschen eine Diskrepanz wahr zwischen den typischen Charakteristika, die Frauen aufweisen (sollten) und den Charakteristika, die erfolgreiche Führungsperson aufweisen (sollten). Diese Einstellung bewirkt zwei Formen von Vorurteilen: a) werden Frauen als weniger geeignet für leitende Positionen eingeschätzt und b) wird Führungsverhalten weniger positiv beurteilt, wenn es von Frauen ausgeübt wird. Man kann diese Theorie stark vereinfachend so formulieren: Eine »richtige « Frau kann nicht führen/ist nicht geeignet für eine berufliche Karriere; andererseits kann eine Frau, die gut führen kann und beruflich Karriere macht, keine »richtige« (vor allem 
keine weibliche) Frau sein. Eagly und Karau (2002) berichten über empirische Studien, die diese Annahmen unterstützen. Im Alltagsleben zeigen sich ebenfalls Belege für die Richtigkeit der Rollen-(In-)Kongruitätstheorie. Ein gutes Beispiel sind öffentliche Urteile über Politikerinnen: Sehr schnell bekam die ehemalige britische Premierministerin Margaret Thatcher den Beinamen »Iron Lady« verpasst. Auch das Studium von Kontakt- und Heiratsanzeigen kann die Theorie untermauern. In solchen Anzeigen scheinen beruflich engagierte und erfolgreiche Frauen ihre Weiblichkeit besonders betonen zu müssen, wenn sie ihre Chancen auf eine Antwort erhöhen wollen. Dazu ein aktuelles Beispiel, wie eine sexklusive" Partnerschaftsvermittlerin seit Jahren beruflich engagierte Akademikerinnen »an den Mann« zu bringen versucht: »Entzückende Mädchenfrau, 32/164, Projektmanagerin, stilvoll, charmant, gut aussehend, mit schönem langem Haar und zierlicher, ideal weiblich proportionierter Figur, selbstbewusst, temperamentvoll, witzig, zuverlässig, emotional, einfühlsam, liebevoll, möchte ihren ,Seelenverwandten s mit Persönlichkeit, Herz und Intellekt für eine gemeinsame Lebensplanung finden« (Der Tagesspiegel vom 20.03.05, Rubrik »Heirat, Bekanntschaft, Partnerschaft«).

Fremdbeurteilungsbiases und Vorurteile bei der Beurteilung karriererelevanter Qualifikationen von Frauen sind mehrfach empirisch nachgewiesen worden. Hierzu gehört die in der Einleitung bereits kurz beschriebene Studie zur Benachteiligung schwedischer Wissenschaftlerinnen bei der Zuteilung von Forschungsstipendien (Wenneras \& Wold 1997). In einer Metaanalyse haben Davison und Burke (2000) die Effekte von Geschlechtsdiskriminierung in simulierten Bewerbungsgesprächen zusammengestellt. Danach neigen nicht nur männliche, sondern auch weibliche Beurteiler zu einer ungünstigeren Beurteilung von Frauen in simulierten Bewerbungs- und Einstellungsstudien. Bühren (2001) führte eine Umfrage unter 103 Lehrstuhlinhabern und Abteilungsleitern chirurgischer Abteilungen an deutschen Universitätskliniken durch. Darin erwies sich ein konservatives Geschlechterrollenmodell als mögliche Ursache für (unbewusste) Diskriminierung von Frauen. Hier zwei Kommentare von Befragten: »Dieser physisch und mental harte Beruf in Kombination mit einer hoch kompetitiven wissenschaftlichen Karriere ist nicht mit Ehe und Familie vereinbar «. Oder: »Einzige Erklärung, warum sich nicht mehr (Frauen) habilitieren: weniger Aggressivität und »Ehrgeiz« (im eigentlichen Wortsinne) bei den Frauen.« (Zitate aus Bühren 2001).

Die Frage ist jedoch nicht nur, ob andere Personen Frauen Führung und Karriere »zutrauen «, sondern auch, ob Frauen selbst der Meinung sind, dass sie Karriere machen können, ohne sich selbst zu verbiegen oder sich selbst »untreu « zu werden. So höre ich häufig von Studentinnen und jungen Akademikerinnen Aussagen wie diese: »Karriere kann ich nur machen, wenn ich mich verbiege«, "Karriere geht auf Kosten meiner Weiblichkeit«, oder: »Ich will nicht männliche Attribute übernehmen, um Karriere machen zu können«. Wenn eine Frau ein sol- 
ches Geschlechterstereotyp verinnerlicht hat, wird es ihr tatsächlich schwer fallen, das eigene weibliche Selbstkonzept mit dem Bild einer beruflichen Karriere zu vereinbaren. Interessanterweise etikettieren Frauen ihr eigenes selbstbewusstes und durchsetzungsfähiges Verhalten, wenn es im familiären Bereich, z. B. gegenüber den Kindern gezeigt wird, nicht als »männlich «.

In diesem Zusammenhang möchte ich einige Ergebnisse aus eigenen Studien vorstellen, die von der Selbstkonzepttheorie der beruflichen Entwicklung inspiriert wurden. Nach dieser Theorie suchen Menschen sich einen Beruf aus, von dem sie glauben, dass sie dafür das richtige Eigenschaftsprofil mitbringen und dass sie in diesem ihr Selbstkonzept verwirklichen können. Welche Persönlichkeitseigenschaften werden als förderlich für eine Karriere im ärztlichen Beruf angesehen? Diese Frage untersuchte ich in einer Studie, an der insgesamt 450 StudienanfängerInnen und PJlerInnen der Freien Universität Berlin teilnahmen (Sieverding 1990). Die Studienteilnehmer sollten anhand einer Liste von Persönlichkeitseigenschaften beurteilen, inwieweit diese förderlich sind, in der Klinik eine Stelle zu bekommen, zu behalten und in der Klinik aufzusteigen. Natürlich können Medizinerinnen auch außerhalb der Klinik Karriere machen, z. B. in einer eigenen Praxis. Notwendig ist dafür in der Regel jedoch ein Facharztabschluss, der nur im Rahmen einer mehrjährigen Tätigkeit an einer Klinik erlangt werden kann. In der Eigenschaftsliste waren zwei Skalen zur Erfassung von Persönlichkeitseigenschaften, die als typischer für Männer oder für Frauen gehalten werden, sowie zwei Skalen zum Leistungsstreben und zur Selbstbehauptung. Die Instrumentalitätsskala des Personal Attributes Questionnaire (dt. Fassung: Runge et al. 1981) enthält aufgabenbezogene Eigenschaften, die als typisch maskuline Persönlichkeitsattribute gelten, wie »selbstsicher «, »durchsetzungsfähig «, »aktiv«, »leicht Entscheidungen fällend«. Die Expressivitätsskala enthält Eigenschaften, die der sozialemotionalen Unterstützung anderer dienen, wie »hilfsbereit «, » einfühlsam « oder »freundlich «. Expressive Eigenschaften gelten als typisch feminine Qualitäten.

Von allen vier Befragtengruppen wurden instrumentelle Eigenschaften als deutlich förderlicher für eine berufliche Karriere im Krankenhaus eingestuft, während expressive Eigenschaften als sehr viel weniger förderlich angesehen wurden (- Abb. 1.15). Das Gleiche galt für die ebenfalls erfassten - nicht näher definierten - Eigenschaften »männlich « und »weiblich «. Das Selbstkonzept wurde anhand der gleichen Eigenschaften erfasst und anschließend ein Diskrepanzscore zwischen Karrierekonzept und Selbstkonzept berechnet. Bei den Studienanfängern gab es in diesem Diskrepanzscore keinen Geschlechtsunterschied, wohl aber bei den Befragten im Praktischen Jahr. Bei den Medizinerinnen am Ende des Studiums war die Diskrepanz signifikant größer als bei ihren männlichen Kommilitonen. Diese größere Diskrepanz war auf zwei Phänomene zurückzuführen. Einerseits hatten die PJlerinnen ein besonders maskulines Karrierekonzept, d. h., sie hatten noch mehr als Studienanfängerinnen und männliche Kollegen das Gefühl, dass feminine 


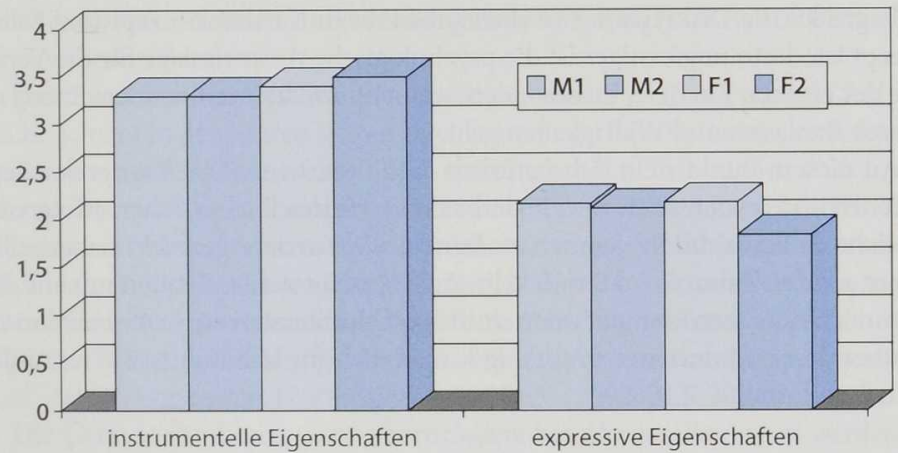

- Abb. 1.15. Subjektives Karrierekonzept bei Medizinstudierenden. Welche Eigenschaften sind förderlich für eine berufliche Karriere im Krankenhaus?

Anmerkungen. Mögliche Werte von 0 (»überhaupt nicht förderlich«) bis 4 (»sehr förderlich«); $n=450 ;$ M1/F1: Männer/Frauen am Anfang des Medizinstudiums, M2/F2: Männer und Frauen am Ende des Medizinstudiums (Quelle: Sieverding 1990)

Eigenschaften in der Klinik überhaupt nicht zählen (『 Abb. 1.15). ${ }^{2}$ Andererseits wiesen die Medizinerinnen am Ende des Studiums ein feminineres Selbstkonzept als die Medizinerinnen zu Beginn des Studiums auf.

Je femininer sich die Medizinerinnen in ihrem Selbstkonzept beschrieben, desto geringer waren auch ihr berufliches Selbstvertrauen, ihre Karrieremotivation sowie ihre Hoffnungen, dass sie ihre Karrierepläne verwirklichen können würden. Ein instrumentelles Selbstkonzept und berufliches Selbstvertrauen (bekannt auch als so genannte »career self-efficacy «) sind jedoch wichtige psychologische Ressourcen für eine berufliche Karriere, wie in der Längsschnittstudie BELA von Abele und Mitarbeiterinnen gezeigt werden konnte (Abele \& Stief 2004 sowie Abele in diesem Band).

Buddeberg-Fischer et al. (2002) untersuchten »Karrierewünsche und Karriereängste« von Medizinstudierenden der Universitäten Basel, Bern und Zürich in Fokusgruppeninterviews. Auch sie fanden bei den Medizinstudentinnen die Diskrepanz zwischen subjektivem Karrierekonzept und Selbstkonzept als innere Barriere: Eine Medizinstudentin formulierte diesen Konflikt so: $\gg$ Da muss ich wirklich tough dahinter sein, meine Ellbogen ausstrecken zu können, meinen Weg gehen und nicht zögern. Und davor habe ich im Moment sicher noch Angst und will es auch nicht eingehen« (Buddeberg-Fischer et al. 2002).

2 So beschrieben einige ihre Erfahrungen in der Klinik, dass sie als "Schwester « angesprochen wurden, wenn sie besonders mitfühlend zu den Patienten waren. 
Je größer die Diskrepanz zwischen subjektivem Karrierekonzept und Selbstkonzept ist, desto ungünstiger ist die psychologische Ausgangslage für die Verfolgung der eigenen Karriere, insbesondere wenn es um die Auseinandersetzung mit äußeren Barrieren und Widrigkeiten geht.

An diesem Punkt stellt sich natürlich die Frage, wie solche Karrierekonzepte entstehen und inwieweit sie modifizierbar sind. Hilfreich ist es sicher, erfolgreiche weibliche Rollenvorbilder kennen zu lernen, die Karriere gemacht haben, ohne »Haare auf den Zähnen « zu entwickeln. Andererseits werden Frauen nur aus dem Dilemma ausbrechen können, wenn sie die geschlechterstereotpye Assoziation von selbstbewusst und durchsetzungsfähig = männlich für sich selbst (wie für andere Frauen) auflösen.

\subsubsection{Die Mutter-Kind-Ideologie}

Die Unvereinbarkeit von Kind und Beruf gilt als die »klassische« Barriere für die Berufstätigkeit und die berufliche Karriere von Frauen. Für die Pionierinnen im Ärztinnenberuf beispielsweise war die Unvereinbarkeit der beiden Rollen explizit; sie wurden mit einer polarisierten Lebensentscheidung, mit einer Entscheidung für eine der Rollen konfrontiert: medizinische Karriere oder Heirat. In manchen Bundesländern wurde verheirateten Ärztinnen die Kassenzulassung verweigert, in anderen mussten sie bei Zulassung Erklärungen unterschreiben, mit der Verpflichtung, bei Heirat auszuscheiden. Nur ein Bruchteil der Ärztinnen, die in den ersten Jahrzehnten dieses Jahrhunderts ihren Abschluss gemacht haben, hat geheiratet oder Kinder bekommen. Dieser Sachverhalt änderte sich in den nachfolgenden Jahrzehnten deutlich, die meisten Ärztinnen heirateten und bekamen Kinder. Allerdings verzichteten diese dann zeitweise oder auch ganz auf die Ausübung ihres Berufes. So waren beispielsweise im Jahr Ende 1987 in der Bundesrepublik Deutschland und West-Berlin 19.631, d. h. 30\% aller approbierten Ärztinnen nicht als Ärztin berufstätig (bei den Männern 16\%). Von diesen war jedoch nur jede sechste, nämlich insgesamt 3200, arbeitslos gemeldet (Sieverding 1990). Berufstätigkeit und Familie lassen sich im ärztlichen Beruf schwerer miteinander vereinbaren $^{3}$ als in anderen Berufsfeldern; die durchschnittliche wöchentliche Arbeitszeit in der Klinik liegt bei 55 Stunden (Stern 1996); durch Überstunden und Bereitschaftsdienste ist zudem die Arbeitszeit schwer kalkulierbar. In der Regel finden Männer leichter eine Partnerin, die bereit ist, für die Familie ihre eigenen berufli-

3 Dies gilt zumindest für Westdeutschland; in der ehemaligen DDR war eine deutlich bessere Vereinbarkeit gegeben, vor allem wegen eines umfangreichen Angebots an staatlichen Krippen, Kindergärten und Ganztagesschulen. In Westdeutschland dagegen gibt es kaum Angebote zur Ganztagesbetreuung. 
chen Ambitionen zurückzustecken oder ganz aufzugeben (s. dazu den Beitrag von Abele in diesem Band).

Das Zurückstellen des beruflichen Engagements zugunsten von Kind und Familie scheint in den letzten Jahren abgenommen zu haben. Inzwischen gibt es in der Bundesrepublik Deutschland den erschreckenden Tatbestand, dass immer mehr Akademikerinnen kinderlos bleiben. So stieg in Westdeutschland die Zahl kinderloser Akademikerinnen (bis zu 40 Jahren) in einem Zehnjahreszeitraum von 32\% im Jahr 1991 auf 42\% im Jahr 2001 (iwd, 2003)! Im gleichen Zeitraum stieg in Deutschland der Frauenanteil an abgeschlossenen Promotionen von 28,5 (1990) auf $36,4 \%$ (2002, dpa-Meldung vom 24.02.05) und der Frauenanteil an abgeschlossenen Habilitationen von 12,9\% (1992) aus 21,6\% (2002; BLK 2004).

Die Gründe für diesen Geburtenrückgang bei Akademikerinnen werden vor allem in den unzureichenden Betreuungsmöglichkeiten für Kinder gesehen. Tatsächlich ist die Betreuungssituation in Westdeutschland im Vergleich zu anderen Ländern äußerst unzureichend. Daneben wirkt jedoch eine weitere psychologische Barriere, die so genannte Mutter-Kind-Ideologie, die vermutlich - zumindest zum Teil - für das Fehlen von Ganztagsbetreuungseinrichtungen in (West-)Deutschland mitverantwortlich ist. Diese Ideologie kann relativ einfach über die Zustimmung zu folgender Aussage gemessen werden: »Ein Vorschulkind leidet, wenn die Mutter berufstätig ist«. Obwohl vielfach widerlegt, hält sich dieses Vorurteil hartnäckig, zumindest im Westen Deutschlands. Dass die Meinungen über diese Aussage auseinander gehen, zeigt sich beispielsweise in einer Studie, in der die Zustimmungsquoten von verheirateten Frauen mit Kindern im Ländervergleich miteinander verglichen wurden (Höllinger 1991). Während im Jahr 1988 in den USA und Großbritannien 43 bzw. 47\% der Befragten der Aussage zustimmten, waren es in Italien $67 \%$, der ehemaligen Bundesrepublik Deutschland 72\%, in Österreich 77\%. Stellt man diesen Zustimmungsquoten ein Maß für die Erwerbsbeteiligung von Frauen gegenüber, zeigt sich ein interessantes Muster: Je höher die Zustimmungsraten zur Mutter-Kind-Ideologie in einem Land war, desto niedriger war dort auch die Erwerbsquote von Frauen im Alter von 30-44 Jahren. Dieses Ergebnis war zu erwarten. Interessanterweise waren in diesen Ländern jedoch gleichzeitig auch die Geburtenraten niedriger (•Tab. 1.6). Die Mutter-Kind-Ideologie scheint somit nicht mit einer höheren Geburtenrate, sondern sogar mit einer niedrigeren Geburtenrate verbunden zu sein!

In der 2003 durchgeführten Online-Umfrage »Perspektive Deutschland « (Kluge et al. 2004) wurde nach möglichen Gründen für die geringe Geburtenrate in Deutschland gefragt. Dass berufstätige Mütter als »Rabenmütter « angesehen werden, wurde zwar nur von $12 \%$ der Befragten als Grund angegeben. Wurde jedoch neutraler gefragt, ob Kinder unter der Berufstätigkeit von Müttern leiden, stimmten immerhin $43 \%$ der westdeutschen Befragten zu. In Ostdeutschland lag die $\mathrm{Zu}$ stimmungsquote nur bei $25 \%$. Die Mutter-Kind-Ideologie ist vor allem ein west- 


\begin{tabular}{|c|c|c|c|}
\hline & $\begin{array}{l}\text { Zustimmungsquote } \\
\text { Mutter-Kind-Ideologie } \\
\text { (1988) }\end{array}$ & $\begin{array}{l}\text { Erwerbsquote der } \\
30 \text { - bis } 44 \text {-jährigen } \\
\text { Frauen (1985) }\end{array}$ & $\begin{array}{l}\text { Geburten } \\
\text { pro } 1000 \\
\text { Bev. (1985) }\end{array}$ \\
\hline USA & $43 \%$ & 72 & 16 \\
\hline Großbritannien & $47 \%$ & 71 & 13 \\
\hline Italien & $67 \%$ & 55 & 10 \\
\hline $\begin{array}{l}\text { Bundesrepublik } \\
\text { Deutschland }\end{array}$ & $72 \%$ & 62 & 10 \\
\hline Österreich & $77 \%$ & 62 & 12 \\
\hline
\end{tabular}

deutsches Phänomen, in der ehemaligen DDR war die gleichzeitige Ausübung der Mutter- wie der Berufstätigenrolle selbstverständlich. So haben vor der Wende nur $0,5 \%$ aller Frauen in Ostdeutschland wegen der Berufstätigkeit auf Kinder verzichtet, während es bei den westdeutschen Frauen 33\% waren (BLK 2004). Und auch heute ist die Kinderlosigkeit unter ostdeutschen Akademikerinnen zwar ebenfalls zunehmend, aber deutlich geringer ausgeprägt als bei den westdeutschen Akademikerinnen (1991 bei 14\%, 2001 bei 17\%, idw 2003).

\subsubsection{Zu bescheidene Selbsteinschätzung und Selbstdarstellung}

Kay Deaux, die in mehreren Studien zunächst Fremdbeurteilungsbiases in der Beurteilung weiblicher Leistung untersucht hatte, formulierte schon Ende der 70er Jahre die Vermutung, dass (zu) ungünstige Selbstbeurteilungen von Frauen ihre Karrierechancen beeinträchtigen können (Deaux 1979).

In einer Fülle von Studien wurden Selbstbeurteilungen im Geschlechtervergleich untersucht, mit dem weitgehend konsistenten Ergebnis, dass Frauen in Leistungssituationen ihre Leistungen unterschätzen (Überblick in Sieverding 2003). Ehrlinger und Dunning (2003) beispielsweise untersuchten die Selbsteinschätzungen der wissenschaftlichen Fähigkeit von College-Studierenden, wobei sie zwischen allgemeinen Selbsteinschätzungen (»chronic self-views«) der wissenschaftlichen Fähigkeit und der Selbsteinschätzung der konkreten Leistung in einem Test unterschieden. Sie konnten zeigen, dass Frauen eine negativere allgemeine Selbsteinschätzung ihrer wissenschaftlichen Fähigkeit besaßen und dass sie auch ihre konkrete Leistung in einem Wissenschaftsquiz negativer beurteilten als Männer, ob- 
wohl sich in der objektiven Leistung im Test keine Geschlechtsunterschiede zeigten. Frauen zeigten im Anschluss an den Test weniger Interesse, an einem Wissenschaftswettbewerb teilzunehmen, in dem interessante Preise zu gewinnen waren. Dabei hing das Desinteresse der Frauen stärker mit ihren Selbsteinschätzungen der Leistungen im Test als mit ihren tatsächlichen Testleistungen zusammen.

Aufschlussreich sind weiterhin Studien, die Selbsteinschätzungen im beruflichen Setting untersuchten. Deaux (1979) erfasste die Selbstbeurteilungen von weiblichen und männlichen Managern in vergleichbaren Positionen und fand eine Reihe von Geschlechtsunterschieden. Männliche Manager beurteilten nicht nur ihre Gesamtleistung als besser, sie schätzten auch ihre Fähigkeiten und ihre Intelligenz höher ein als weibliche Manager. Männliche Manager attribuierten darüber hinaus ihren Erfolg mit höherer Wahrscheinlichkeit auf ihre Fähigkeiten als weibliche Manager. In der Fremdbeurteilung durch die (männlichen) Supervisoren fanden sich jedoch nur geringfügige Unterschiede; insbesondere wurden weibliche und männliche Manager in Hinsicht auf ihre allgemeinen Leistungen, Fähigkeiten und Motivation nicht als unterschiedlich beurteilt. Auch in einer neueren Studie zeigten sich noch Geschlechtsunterschiede in der Selbstbeurteilung von Managern: Managerinnen attribuierten ihren Erfolg weniger stark auf ihre Fähigkeiten als Manager (Rosenthal et al.1996).

Lindeman et al. (1995) verglichen die Selbstbeurteilungen von männlichen und weiblichen Bankkaufleuten mit ihrer objektiven Leistung. Als Indikator der objektiven Leistung wurde die Verkaufsleistung in einem Dreimonatszeitraum herangezogen. Die Akkuratheit der Selbsteinschätzung wurde definiert als die Differenz zwischen der (jeweils standardisierten) Verkaufsleistung und Selbsteinschätzung. Sie fanden, dass Männer sich selbst positiver als Frauen beurteilten; zwei Drittel der Männer wurden als »Selbstüberschätzer« diagnostiziert, während Frauen sich gleichmäßig auf die Gruppen der »Selbstunterschätzer«, »akkurate Selbsteinschätzer« und »Selbstüberschätzer « verteilten.

Wie sieht es mit Selbsteinschätzung und Selbstdarstellung in Bewerbungsgesprächen aus? Diese Frage wurde in einer Studie an der Freien Universität Berlin untersucht (Sieverding 2000, 2003). Ein Vorstellungsgespräch ist in der Regel eine notwendig zu überwindende »Hürde« auf dem Weg zur gewünschten Stelle oder zur beruflichen Position dar. Der Eindruck, den ein Bewerber oder eine Bewerberin in dieser zeitlich sehr begrenzten Situation macht, gibt häufig den entscheidenden Ausschlag über Anstellung oder Nichtanstellung und damit über wichtige Karriereoptionen. In einem solchen Job-Interview, in dem man in der Regel unmittelbar mit den Mitbewerbern der engeren Wahl verglichen wird, ist es besonders wichtig, die eigenen Stärken zu kennen und entsprechend nach außen darstellen zu können. Um jedoch selbstbewusst auftreten zu können, ist eine realistische Einschätzung der eigenen Fähigkeiten notwendig. Ziel der Studie war es, potentielle Selbstbeurteilungsbiases bei Frauen und Männern in einer Bewerbungssituation zu untersuchen. 
Die Stichprobe bestand aus 37 Männern und 37 Frauen aus verschiedenen Studienfächern, für die eine berufliche Bewerbung in absehbarer Zeit aktuell war. Die meisten Probanden befanden sich im Hauptstudium, kurz vor Abschluss des Studiums, einige hatten ihr Studium bereits abgeschlossen (4 Männer, 7 Frauen). Die Probanden waren zwischen 22 und 34 Jahre alt; das Durchschnittsalter lag bei 26 Jahren. Die Teilnehmer der Studie mussten im Labor in Einzelversuchen verschiedene Aufgaben absolvieren, wie sie typisch für Bewerbungssituationen sind: Einen schriftlichen Leistungstest, einen Vortrag zur Selbstdarstellung der beruflichen Qualifikation sowie ein standardisiertes Bewerbungsinterview. Vortrag und Bewerbungsinterview wurden videographiert. Nach jeder Untersuchungsphase sollten die Teilnehmer einschätzen, wie erfolgreich sie sich in der vergangenen Phase gefühlt hatten. Dabei konnten Werte von 0 (»überhaupt nicht erfolgreich) bis 9 (»sehr erfolgreich») angekreuzt werden. Nach Beendigung der Aufgabenphasen wurden die Probanden in einer schriftlichen Nachbefragung gebeten, sich zum Erleben der verschiedenen Abschnitte der Untersuchung zu äußern. Das Verhalten der Bewerber und Bewerberinnen wurde später von zwei Expertinnen, einer Diplompsychologin und einer Ärztin, anhand eines Videoausschnittes aus dem Bewerbungsinterview beurteilt.

Geschlechtsunterschiede zeigten sich in der Selbsteinschätzung wie in der Selbstdarstellung. Zunächst zur Selbsteinschätzung: Frauen schätzten sich in allen Phasen des Versuchs als deutlich weniger erfolgreich ein. Dabei trat dieser Geschlechtsunterschied schon vor Beginn der eigentlichen Untersuchung auf, d. h., bevor die eigentlichen Aufgaben bearbeitet wurden. Die Diskrepanz zwischen männlichen und weiblichen Selbsteinschätzungen betrug im Durchschnitt ca. 2 Skalenpunkte! (• Abb. 1.16).

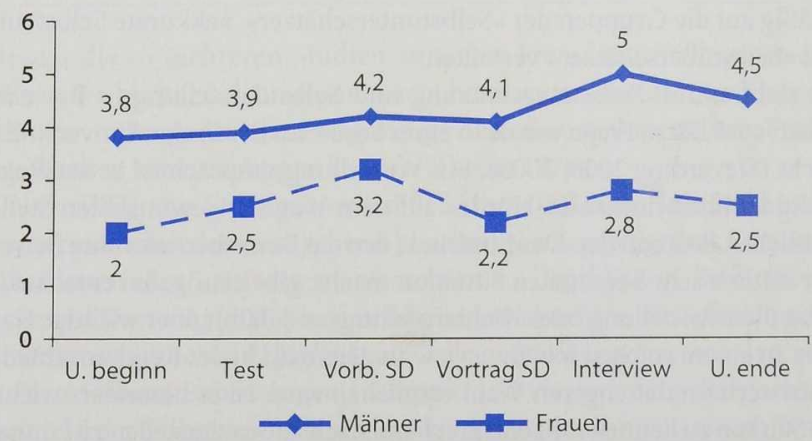

- Abb. 1.16. Selbsteinschätzung als serfolgreich« im Job-Interview Anmerkungen. Arithmetische Mittelwerte; $n=74$; mögliche Werte von 0 (»überhaupt nicht erfolgreich«) bis 9 (»sehr erfolgreich«); SD = Selbstdarstellung (Quelle: Sieverding 2003) 
Interessant ist nun die Frage, ob die Geschlechtsunterschiede in der Selbsteinschätzung als erfolgreich auf eine Selbstunterschätzung der Frauen, auf eine Selbstüberschätzung der Männer oder auf beide Phänomene zurückzuführen sind. Deshalb wurden die Selbsteinschätzungen mit den objektiven Leistungen im schriftlichen Leistungstest sowie mit Fremdeinschätzungen des Verhaltens im Bewerbungsinterview verglichen. In den verschiedenen Untertests des Leistungstests fanden sich keine signifikanten Geschlechtsunterschiede. Männer und Frauen erreichten hier im Durchschnitt vergleichbare Werte. In den Fremdeinschätzungen dagegen wurden die Männer ebenfalls als erfolgreicher eingeschätzt als die Frauen (die Mittelwerte betrugen $M=4,7$ für die männlichen und $M=3,7$ für die weiblichen Bewerber), der Geschlechtsunterschied in den Fremdeinschätzungen war jedoch deutlich geringer als der in den Selbsteinschätzungen (• Abb. 1.16). Im Vergleich zur erreichten Punktezahl in den Leistungstests und zur Fremdbeurteilung eines Videoausschnittes aus dem Bewerbungsinterview lag bei Frauen eine eindeutige Selbstunterschätzung vor. Bei den Selbstbeurteilungen der Männer zeigte sich im Vergleich zum Leistungstest eine gewisse Selbstüberschätzung, im Vergleich zur Fremdbeurteilung dagegen eine realistische Selbsteinschätzung.

Auch in der Selbstdarstellung gab es Geschlechtsunterschiede. Die Studienteilnehmer hatten die Aufgabe, einen 5-minütigen Bewerbungsvortrag (Selbstdarstellung der beruflichen Qualifikationen) zu halten und waren aufgefordert, diese 5 Minuten auch zu nutzen. Nur jeder vierte Proband $(n=18)$ nutzte die vorgegebene Zeit entsprechend der Instruktion voll aus. Das Spektrum reichte von 50 bis 300 Sekunden, der arithmetische Mittelwert lag bei $M=196$ Sekunden $(\mathrm{SD}=84)$. Es zeigte sich ein hochsignifikanter Geschlechtsunterschied: Männer redeten im Durchschnitt fast eine Minute länger als Frauen $(M=222$ versus $M=170$; Sieverding 2000).

In einer multiplen Regressionsanalyse wurde abschließend berechnet, welche Faktoren die Fremdeinschätzung als »erfolgreich" vorhersagen können: Ist es das biologische Geschlecht, ist es die Leistung im schriftlichen Test, ist es die Selbsteinschätzung als »erfolgreich «, ist es die Dauer der Selbstdarstellung? Welche dieser Faktoren können vorhersagen, inwiefern ein Bewerber oder eine Bewerberin als serfolgreich « eingestuft wird? Das Ergebnis dieser Analyse ist in Tab. 1.7 dargestellt.

Das biologische Geschlecht der BewerberInnen spielte keine signifikante Rolle mehr für die Fremdbeurteilung, wenn die Selbsteinschätzung als erfolgreich und die Dauer der Selbstdarstellung berücksichtigt wurden. Erfolgreich wurden solche BewerberInnen eingeschätzt, die die Zeit für die Selbstdarstellung möglichst lange ausgenutzt hatten und die sich selbst als erfolgreich eingeschätzt hatten! Ein besonders gutes Abschneiden in einem Teil des schriftlichen Leistungstests war sogar negativ mit der Fremdeinschätzung als erfolgreich assoziiert, dieses Ergebnis soll nicht überinterpretiert werden, verweist jedoch auf die Tatsache, dass Selbsteinschätzung und Selbstdarstellung der Bewerber wichtiger war als die objektive Leis- 
- Tab. 1.7. Multiple Regression zur Vorhersage der Fremdeinschätzung als »erfolgreich« im Bewerbungsinterview

\begin{tabular}{|c|c|c|c|}
\hline Prädiktor & R2cum & Beta & $\mathrm{t}$ \\
\hline Dauer der Selbstdarstellung & 0,19 & 0,31 & $2,7^{* *}$ \\
\hline Selbsteinschätzung als erfolgreich & 0,25 & 0,35 & $2,9^{* *}$ \\
\hline Abschneiden im schriftlichen Leistungstest ${ }^{a}$ & 0,29 & 0,22 & $-2,0^{*}$ \\
\hline \multicolumn{4}{|c|}{$\begin{array}{l}\text { Anmerkungen. } R^{2} \times 100=\text { aufgeklärte Varianz in } \% \text {. } \\
\text { a Würfeltest des Intelligenz-Struktur-Test; }{ }^{*} p<0,05 ;{ }^{* *} p<0,01 \text { (Quellen: Sieverding 2000, } \\
\text { 2003). }\end{array}$} \\
\hline
\end{tabular}

tung. Die Ergebnisse der vorliegenden Studie unterstützen somit die These eines Vorurteils gegenüber weiblichen Bewerbern - zumindest für weibliche Beurteiler - nicht. Vielmehr unterstützen die hier erzielten Befunde die Hypothese, dass Potentialunterschätzungen nicht nur bei externen Beurteilern eine Rolle spielen können, sondern auch bei der Beurteilung der eigenen Person. Meine These ist, dass auch im Berufsalltag viele Frauen ihr eigenes Potential unterschätzen, und dass eine solche Unterschätzung gerade in einer Bewerbungssituation zur »self-fulfilling prophecy werden kann. Tatsächlich wurden ja die weiblichen Kandidaten als signifikant weniger erfolgreich beurteilt im Vergleich zu den männlichen Kandidaten, und zwar von weiblichen Beurteilern.

Es ist zu vermuten, dass viele Frauen an sich selbst ein unrealistisch hohes Anspruchsniveau anlegen, vor dem dann ihre Selbstbeurteilung - im Vergleich zu ihren männlichen Konkurrenten - zu schlecht ausfällt. Hennig und Jardim (1987) haben dieses Phänomen damit erklärt, dass Männer die Welt häufiger so sehen, wie sie ist und Frauen sie sehen, wie sie sein sollte. Viele Frauen in einer Bewerbungssituation scheinen sich auch so zu sehen, wie sie - ihrer Meinung nach - sein sollten, und resignieren angesichts einer zu hohen Erwartungshaltung. Das negative psychologische Potential solcher Selbsteinschätzung ist aus den Aufsätzen in der Nachbefragung zu erkennen. Während Männer häufig im Nachhinein die Situation als »Herausforderung " oder als »Chance « beschreiben, bewerten nicht wenige Frauen die Situation als »Bedrohung « oder » Verlust «, ihr eigenes Verhalten als » Versagen «. Dazu einige Beispiele zunächst von männlichen Probanden:

„Während des Tests wurde mir aber wieder verstärkt klar, wie viel Engagement nötig sein wird, später wirklich mal einen interessanten Job zu kriegen. Besonders das präzise Ausformulieren meiner Gedanken und Antworten machte mir mehr Schwierigkeiten, als ich mir selbst gewünscht hätte. Andererseits machte es mir aber Freude, eine später wohl wirklich auftretende, Stress'-Situation jetzt schon durchspielen zu können." (Pb 01204, männlich) 
"Habe ganz guten Eindruck auf den Interviewer gemacht. Hätte vielleicht noch ein Späßchen mehr machen können, um die Situation aufzulockern. Seine eigenen Vorteile anzupreisen ist total ungewohnt, habe Hemmungen dabei (Angeber!) ... Fühle mich aber ziemlich gut nach der Untersuchung. Ob ich dem Interviewer woh/ sympathisch bin? Habe ich zu sehr meine Einfüh/samkeit betont? (Pb 01205, männlich)

Dazu im Vergleich zwei Beispiele von Einschätzungen weiblicher Probanden:

„Es ist eine ziemlich stressige Situation, wenn Fragen zur persönlichen Eignung zu beantworten sind, aber auch, wenn ein Kurzvortrag gefordert ist, indem man seine Eignung, seine Qualitäten möglichst gut herausstellen soll. Ich fühle mich in solchen Situationen immer ziemlich mies, habe das Gefühl, mich verkaufen zu müssen und das liegt mir gar nicht. Irgendwie ist es für mich etwas würdelos. Besser finde ich die Möglichkeit, sich im Gespräch äußern zu können oder in einem Test bestimmte Fertigkeiten nachweisen zu müssen. Ich glaube, ziemlich unsicher gewirkt zu haben. Obwohl ich mir vornehme, nicht als Verliererin zu empfinden, geht es mir so, dass ich mir von vornherein wenig Chancen gebe. Wichtig fände ich für solche Personalbewerbungsgespräche, dass eine zwanglose Atmosphäre herrscht. Sonst profitieren von der Drucksituation nur die Selbstdarsteller-Typen.«(Pb 02233, weiblich)

»Für mich ist die Situation schon unangenehm, vor allem die Tatsache, gezwungenermaßen zu überlegen, warum eigne ich mich.

- Letztlich: was ist an mir gut/gut genug/besser als an anderen?

- Das, Ich-armer-Hanswurst-Gefühl, bzw. ich kann doch schon etwas, doch wie preise ich mich an?

- Erschreckend empfinde ich die Tatsache oder den Eindruck, mich selbst nicht präsentieren zu können, den Bauch fast klopfen zu fühlen ...

- Die vorausschauende Unsicherheit nimmt mir den Atem bzw. macht mir deutlich, wie dringend eine solche Situation zu trainieren wäre...

- Ich fühle mich so gescheitert bzw. ein Versager, da selbst die Kamera, mich anguckt' und die Vorstellung dessen, was einen in einem solchen Gespräch erwartet, ist somit schon jetzt grässlich ... "' (Pb, 02240, weiblich).

Noch im Nachhinein verurteilten sich einige der Frauen, weil sie ihren hohen Erwartungen nicht gerecht worden waren. Hier handelt es sich um eine destruktive Selbsteinschätzung, die einen ungünstigen Einfluss auf das Verhalten in nachfolgenden Situationen haben kann. Ein solcher Übertragungseffekt wird im letzten Zitat angedeutet. Wenn Frauen ihre Selbstdarstellung und damit auch ihre Chancen in einem Bewerbungsgespräch verbessern wollen, erscheint eine realistischere Selbsteinschätzung eine notwendige (wenn auch wahrscheinlich nicht hinreichende) Voraussetzung. Es scheint so zu sein, als ob manche Frauen eine selbstbewusste Selbstdarstellung geradezu als unwürdig oder als »unweiblich « ansehen, nicht zu 
vereinbaren mit dem traditionellen Weiblichkeitsideal der bescheidenen, sich im Hintergrund haltenden Frau. Die Rollen-(In-)Kongruitätstheorie kann auch dieses Phänomen erklären.

\subsubsection{Ausblick}

In diesem Beitrag wurde versucht zu zeigen, wie überholte Geschlechterrollen und -stereotype Frauen einengen und ihre berufliche Karriere behindern oder sogar verhindern können. Diese Geschlechterkonstrukte wirken gewissermaßen doppelt: einerseits von außen, durch die Erwartungen, die unsere soziale Umwelt an uns richtet. Verhalten wir uns nicht entsprechend diesen Erwartungen, haben wir mit mehr oder weniger starken Sanktionen zu rechnen. Eine verbreitete Sanktion ist die, Frauen, die sich nicht erwartungskonform verhalten, die "aus der Rolle« fallen, ihre Weiblichkeit abzusprechen. Andererseits wirken die Geschlechterkonstrukte jedoch auch von innen, und dieser Mechanismus ist besonders effektiv. Je mehr wir geschlechterstereotpye Überzeugungen über »angemessene « oder »unangemessene "Eigenschaften von Frauen (und Männern) verinnerlicht haben, desto schwerer wird es uns fallen, gegen äußere Barrieren anzugehen. Umgekehrt: je mehr wir uns innerlich freigemacht haben von Vorschriften, wie wir "als Frau s sein sollten - und damit schließe ich ausdrücklich auch Erwartungen ein, die »unter Frauen« kursieren -, Vorschriften, wie wir denken, fühlen, handeln sollten, desto eher wird es uns gelingen, unseren Weg erfolgreich zu gehen.

Was kann helfen, solche äußere wie innere Barrieren zu überwinden? Hilfreich sind: der Aufbau und die aktive Nutzung formeller und informeller Netzwerke von beruflich engagierten Frauen, die aktive Suche nach Rollenvorbildern und Mentorinnen, die Erarbeitung einer realistischen Selbsteinschätzung und Selbstdarstellung (u. U. auch in entsprechenden Workshops), die strategische Karriereplanung, bei der je nach Bedarf und Situation durchaus auch auf professionelles Coaching zurückgegriffen werden sollte. Und, nicht zuletzt, sollten Frauen sich ab und zu vor Augen führen, wenn es um ihre eigene berufliche Karriere geht: „Bescheidenheit ist eine Zier, doch weiter komm ich ohne ihr! «

\section{Literatur}

Abele A (1997) Geschlechtsrollen, Geschlechtsrollenorientierungen und Geschlechterstereotype im Wandel. In E. Liebau (Hrsg.), Das Generationenverhältnis. Über das Zusammenleben in Familie und Gesellschaft. Juventa, Weinheim

Abele-Brehm AE, Stief M (2004) Die Prognose des Berufserfolgs von Hochschulabsolventinnen und -absolventen. Befunde zur ersten und zweiten Erhebung der Erlanger Längsschnittstudie BELA-E. Z Arbeits- und Organisationspsychologie 48: 4-16

Alfermann D (1996) Geschlechterrollen und geschlechtstypisches Verhalten. Kohlhammer, Stuttgart 
Bless H, Bohner G, Chassein B, Kittel C, Kohlhoff A, Nathusius K, Schüssler G, Schwarz N (1992) Hausmann und Abteilungsleiterin. Die Auswirkungen von Geschlechtsrollenerwartungen und rollendiskrepantem Verhalten auf die Zuschreibung von Persönlichkeitseigenschaften. Z Sozialpsychol 23: 16-24

Bühren A (2001) UM-Denken ist gefragt auf dem Weg zur Medizin des 21. Jahrhunderts. Ärztin 48: 4-5

Bund-Länder-Kommission für Bildungsplanung und Forschungsförderung (2004). Frauen in der Medizin - Ausbildung und berufliche Situation von Medizinerinnen. Materialien zur Bildungsplanung und zur Forschungsförderung (Heft 117). Verfügbar unter: http://www. blk-bonn.de/papers/heft117.pdf

Buddeberg-Fischer B, Illes C, Klaghofer R (2002) Karrierewünsche und Karriereängste von Medizinstudierenden - Ergebnisse von Fokusgruppeninterviews mit Staatsexamenskandidatinnen und -kandidaten. Das Gesundheitswesen 64: 353-362

Davison HK Burke MJ (2000) Sex discrimination in simulated employment contexts: A metaanalytic investigation. J Voc Behav 56: 225-248

Deaux K (1979) Self-evaluations of male and female managers. Sex Roles 5: 571-580

Deaux K, Major B (1987) Putting gender into context: An interactive model of gender-related behavior. Psychological Rev 94: 369-389

Eagly AH (1987) Sex differences in social behavior: A social-role interpretation. Lawrence Erlbaum, Hillsdale, N.J.

Eagly A-H, Karau SJ (2002) Role congruity theory of prejudice toward female leaders. Psychological Rev 109: 573-598

Eckes T (1997) Geschlechterstereotype: Frau und Mann in sozialpsychologischer Sicht. Centaurus, Pfaffenweiler

Ehrlinger J, Dunning D (2003) How chronic self-views influence (and potentially mislead) estimates of performance. J Personal Soc Psychol 84: 5-17

Hennig M, Jardim A (1987) Frau und Karriere. Rowohlt, Reinbek

Höllinger F (1991) Frauenerwerbstätigkeit und Wandel der Geschlechtsrollen im internationalen Vergleich. Kölner Z Soziol Sozialpsychol 43: 753-771

iwd (2003) Informationsdienst des Instituts der deutschen Wirtschaft Köln Nr. 38 vom 18. September 2003. Verfügbar unter: http://www.iwkoeln.de

Kluge J, Osterkorn T, Laurent S, Schächter M (2004) Projektbericht Perspektive Deutschland 2003/2004. Verfügbar unter: www.perspektive-deutschland.de

Massachusetts Institute of Technology (1999). A study on the status of women faculty in science at MIT. Verfügbar unter: http://web.mit.edu/fnl/women/women.html

Lewin K (1969) Grundzüge der topologischen Psychologie. Huber, Bern

Lindeman M, Sundvik L, Rouhiainen P (1995) Under- or overestimation of self? Person variables and self-assessment accuracy in work settings. J Soc Behav Personal 10: 123-134

Parsons T, Bales RF (1955) Family. Socialization and interaction process. Routledge \& Kegan, London

Rosenkrantz P, Vogel S, Bee H, Broverman I, Broverman DM (1968) Sex-role stereotypes and self-concepts in college students. J Consult Clin Psychol 32: 287-295

Rosenthal P, Guest D, Peccei R (1996) Gender differences in managers' causal explanations for their work performance: A study in two organizations. J Occup Organ Psychol 69: $145-151$ 
Runge TE, Frey D, Gollwitzer PM, Helmreich RL, Spence JT (1981) Masculine (instrumental) and feminine (expressive) traits. A comparison between students in the United States and West Germany. J Cross-Cultural Psychol 12: 142-162

Schmid G, Brzinsky C (2003) Individualisierung und Arbeitsmarkt - Frauen »drängen « auf den Arbeitsmarkt. Überblicksvorlesung "Politik und Wirtschaft «an der FU Berlin, WS 2003/2004. Verfügbar unter: http://www.wz-berlin.de/ars/ab/pdf/vorlesung_praesentation_040112. pdf

Sczesny S (2003) Führungskompetenz: Selbst- und Fremdwahrnehmung weiblicher und männlicher Führungskräfte. Z Sozialpsychol 34: 133-145

Sieverding M (1990) Psychologische Barrieren in der beruflichen Entwicklung von Frauen Das Beispiel der Medizinerinnen. Enke, Stuttgart

Sieverding M (1992) Wenn das Kind einmal da ist ... Die Entwicklung traditionellen Rollenverhaltens bei Paaren mit ursprünglich egalitären Rollenvorstellungen. In Brüderl L, Paetzold B (Hrsg) Frauenleben zwischen Beruf und Familie. Juventa, Weinheim

Sieverding M (2000) »Alle wahren Gefühle verbergen und mit fester Stimme und wohlformulierten Sätzen glänzen!« - Die Bedeutung von Selbstdarstellungsregeln im Bewerbungsinterview. Z Arbeits- und Organisationspsychol 44: 152-156

Sieverding M (2003) Frauen unterschätzen sich: Selbstbeurteilungs-Biases in einer simulierten Bewerbungssituation. Z Sozialpsychol 34: 147-160

Stern K (1996) Ende eines Traumberufs? Lebensqualität und Belastungen bei Ärztinnen und Ärzten. Waxmann, Münster

Wenneras C, Wold A (1997) Nepotism and sexism in peer-review. Nature 387: 341-343

Williams JE, Best DL (1990) Measuring sex stereotypes: A multination study. Sage, Beverly Hill 